\title{
BARRIER CREAMS AND THEIR EVALUATION
}

\author{
BY
}

\author{
W. M. CUMMING, M. C. CAMERON, E. B. CUMMING, and M. C. MACRAILD \\ From the Royal Technical College, Glasgow
}

(RECEIVED FOR PUBLICATION, MARCH 22, 1947)

In view of the recent publication by Sadler and Marriott (1946) on the evaluation of barrier creams, it may be of interest to describe some work carried out during the 1939-45 war. Whilst the work herein described was mainly concerned with explosives, it illustrates the line of attack which might be adopted in dealing with one aspect of the prevention of industrial dermatitis. In the earlier years of the war some anxiety was expressed with regard to the extent of dermatitis in certain factories dealing with explosives. Whilst the highest incidence of dermatitis occurs with tetryl, other nitrobodies, such as T.N.T., are not above suspicion. Tetryl dermatitis has been ascribed by some authorities to a mechanical effect produced by the acicular crystalline form of this product in similarity to the dermatitis produced in many industrial operations. Other authorities claim that the effect is due to absorption of the tetryl by the epidermis of the skin. Experience indicates that the latter is the more likely cause, for dermatitis is often frequent where the material is not in the crystalline form. Tetryl differs from T.N.T. in that the former was prepared finally in dry form whereas T.N.T. was handled mostly molten before being mixed with other substances. Tetryl dermatitis was much more frequent, therefore, in filling than in explosives factories, where in the great majority of operations it was handled wet.

A paper by Witkowski and others (1942) quotes results of an enquiry made at an American factory where 1,258 operatives were employed on tetryl : 944 suffered from dermatitis, though only three of the cases required hospital treatment. In order to control the amount of dust they recommended that the crystalline tetryl should be blended with stearic acid and that granular tetryl (still crystalline but of different form) should be blended with graphite. The authors of that paper developed a barrier, full details of which are not given, and claim that its use cut down the weekly incidence of dermatitis by 50 per cent.
Attention may also be drawn to a paper by Swanston (1943), in which statistics are given of dermatitis in this country. In June, 1942, 3.3 per cent. of the total contact workers were reported as cases of dermatitis, of which one quarter were off work and receiving compensation. In May, 1943, the figure was reduced to 1.5 per cent. Of the total incidence, tetryl dermatitis represents more than one half, mercury fulminate approximately one-third, the remainder being caused by T.N.T., anmonium nitrate, shellac, picric shellite, lead azide, etc. For all practical purposes, the irritant explosives are mercury fulminate and tetryl. T.N.T. may be added not so much because of its high incidence, but rather on account of the severity of the dermatitis produced by it. Dust seems to be the cause of irritation in the majority of cases.

\section{The Present Inquiry}

Early in the war a questionnaire was sent to all medical officers at factories in the hope of gaining some precise knowledge about the incidence of dermatitis and experience of the use of barriers. A statistical study was made difficult by the fact that the quantity of explosives manufactured and handled varied in different factories. Furthermore, at one stage in the war there was a large influx of female labour. In some factories barriers were already in use, while in others the most important factor controlling dermatitis was claimed to be personal hygiene.

From the results of the questionnaire it was possible to make the following deductions: (1) women are more disposed to use barriers than men, and the percentage of dermatitis amongst the latter is higher; (2) face and hands are involved, and contact with air-borne and dry solid explosives seems to be the main cause ; (3) barriers are used at least twice per eight-hour shift.

It would appear therefore, that the case for the use of barriers is justified. In connexion with tetryl dermatitis it is worth noting that the mechanism of 
the interaction is believed to be due to the effect of light on the tetryl once it has gained attachment to the skin surface. Brownlie and Cumming (1946) have shown that tetryl is quantitatively converted into a well-known strongly dermatitic agent, methyl picramide, on exposure for a few hours to ultraviolet irradiation, and that the product is ultimately decomposed by ultra-violet light. Support is given to this work by the fact that tetryl dermatitis reaches its highest incidence in about sixteen days and is claimed to be more prevalent in hot sunny weather. Moreover, it suggests that ultra-violet irradiation of the skin when odema has become apparent would be beneficial.

In order to assess the amount of nitrobody dust and vapour in the atmosphere of explosives factories, methods have been worked out (Cumming and Wright, 1945). As a result of frequent surveys it was possible to improve considerably the indoor atmospheric conditions by modifications in ventilation etc., thereby reducing the risk of contamination of the skin during normal working operations.
Barriers in Use at the Time of the Survey

At this stage it was decided to test the barriers which were already in use at certain factories. To do this it was necessary to devise a simple test. This consisted in applying the barrier to the skin of the forearm after thorough cleansing of the surface with soap and warm water, and drying. The barrier was rubbed in to the skin so as to give a uniform film. The same operatives conducted all tests in the first instance so as to eliminate, as far as possible, the personal element. Preliminary tests on a large number of persons proved that the thickness of film normally applied to the skin is remarkably constant and varied slightly from $20 \mu$. When the film had been thus produced, powdered T.N.T. or tetryl was dusted on to the surface, the surplus shaken or blown off, and the arm exposed to the atmosphere for one to three hours under normal working conditions. After this exposure, the coating on the skin was removed by thorough washing in warm water and soap. The surface of the skin was not dried, nor touched in any other way. A swab of cotton wool moistened with sodium sulphite solution was then rubbed over the surface. If the cotton wool took on a pink colour, the test was taken to be positive, namely, that the

TABLE 1

RESULTS OF TESTS ON BARRIER CREAMS IN USE AT CERTAIN FACTORIES

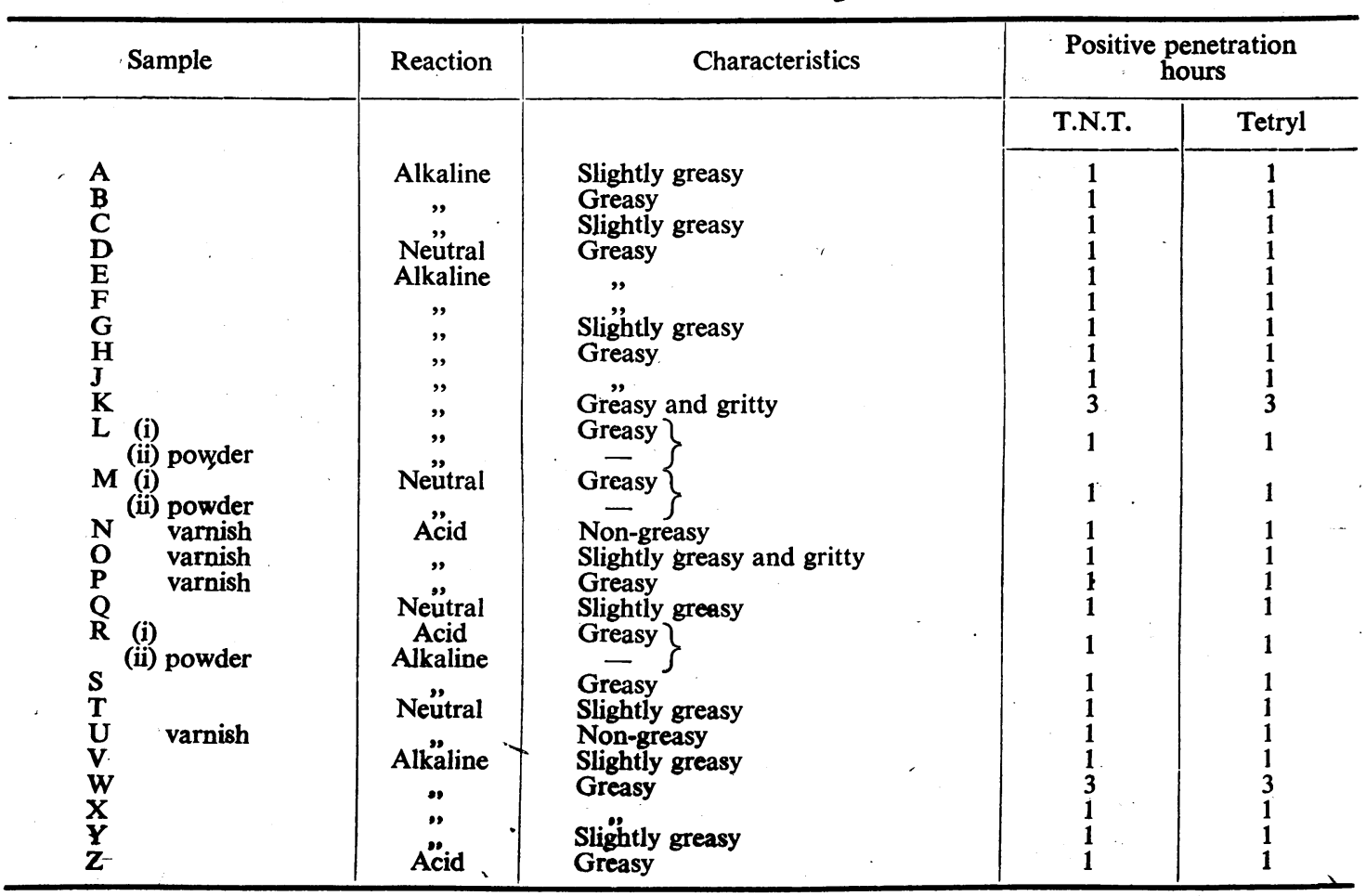


TABLE 2

SOLUBILITIES G/100 G. SOLVENT

\begin{tabular}{|c|c|c|c|c|}
\hline Substance & $\begin{array}{l}\text { T.N.T. } \\
\text { at } 35^{\circ} \text { C. } \\
\text { or temp. stated }\end{array}$ & $\begin{array}{c}\text { Tetryl } \\
\text { at } 35^{\circ} \mathrm{C} \text {. } \\
\text { or temp. stated }\end{array}$ & $\begin{array}{l}\text { Picric Acid } \\
\text { at } 35^{\circ} \mathrm{C} \text {. } \\
\text { or temp. stated }\end{array}$ & $\begin{array}{c}\text { Hexamine } \\
\text { at } 35^{\circ} \mathrm{C} \text {. } \\
\text { or temp. stated }\end{array}$ \\
\hline $\begin{array}{l}\text { Liquid Paraffin } \\
\text { Glycerine } \\
\text { Castor oil } \\
\text { Paraffin wax } \\
\text { Cocoa butter } \\
\text { Lanoline } \\
\text { Lanette wax } \\
\text { Palmitic acid } \\
\text { Stearic acid }\end{array}$ & $\begin{array}{l}0.01 \\
0.10 \\
0.50 \\
0.4\left(58^{\circ}\right) \\
0.6 \\
2.7\left(80^{\circ}\right) \\
0.9\left(80^{\circ}\right) \\
1.0\left(64^{\circ}\right) \\
1.0\left(70^{\circ}\right)\end{array}$ & $\begin{array}{l}0.01 \\
0.2 \\
3.4 \\
0.04\left(65^{\circ}\right) \\
0.3 \\
0.4\left(65^{\circ}\right) \\
0.4\left(75^{\circ}\right) \\
0.3\left(65^{\circ}\right) \\
0.3\left(70^{\circ}\right)\end{array}$ & $\begin{array}{c}\text { Negligible } \\
0 \cdot 40 \\
0 \cdot 10 \\
0.04\left(60^{\circ}\right) \\
0 \cdot 1 \\
2 \cdot 6\left(80^{\circ}\right) \\
0 \cdot 4\left(80^{\circ}\right) \\
\text { Negligible }\left(65^{\circ}\right) \\
\text { Negligible }\left(20^{\circ}\right)\end{array}$ & $\begin{array}{l}0.08 \\
4.10 \\
1.9 \\
0.16\left(65^{\circ}\right) \\
0.48 \\
23.2\left(65^{\circ}\right) \\
2.3\left(75^{\circ}\right) \\
13.7\left(75^{\circ}\right) \\
11.3\left(70^{\circ}\right)\end{array}$ \\
\hline
\end{tabular}

nitro-compound had penetrated through the barrier to the skin surface. If any doubt arose, the test was again repeated in duplicate. When a barrier incorporated the use of a face powder on a vanishing cream ground, both were applied in the customary manner before the nitrobody was dusted on to the surface.

In addition, the barriers were tested for reaction to litmus and the characteristics (greasiness etc.) were agreed upon by four independent observers after application to the skin and rubbing on the surface.

The results of these tests are given in Table 1.

The following conclusions may be drawn.

(a) All barrier creams examined were greasy or slightly greasy in character. The only non-greasy. types occurred in the skin varnish group.

(b) Sixty-five per cent. were alkaline in reaction and, of the remaining 35 per cent., less than half were acid in reaction, these almost invariably occurring in the skin varnishes.

(c) With only two exceptions, the barriers were penetrated by both T.N.T. and tetryl in one hour. These two barriers were, however, penetrated in three hours-by both nitrobodies.

It is clear from the results quoted that none of the barriers in use at the time was efficient. Protection must be given for some hours before their efficiency can be judged to be satisfactory from the practical point of view.

\section{The Ideal Barrier}

The ideal barrier would be a material of the appropriate physical form and composition in which the nitrobodies encountered would be insoluble, and the degree of absorption of the barrier by the skin would be at a minimum. As stated above, the thickness of film produced was found to be about $20 \mu$, which is in accord with the figure mentioned by Sadler and Marriott. The particle size of tetryl, as of many other dusts, which is most physiologically active is $5 \mu$ and under (Bedford and Warner, 1942). No material of this size could, therefore, penetrate the film to the skin surface. For safety the consistency of the barrier should be such as to allow of a film of this order of thickness being obtained in the normal method of application.

It was impossible to determine the solubility of the nitrobodies directly in the barrier cream, but determinations were made in the components which are known to be the main basis of creams and vanishing creams. The results are given in Table 2 . In addition to T.N.T. and tetryl this includes picric acid and hexamine, since in the questionnaire referred to above jt was suspected in one or two cases that dermatitis had been caused by these compounds. Mercury fulminate is not included in the table, as only in lanoline did it show any detectable solubility.

The components were treated with excess of the dermatitic agent and maintained at a constant temperature for one hour. After separation of the excess, acetone (for tetryl) or alcohol (for T.N.T.) was added to dissolve, and the coloration produced with alkali, compared against a standard, gave the required result. In the case of picric acid, potassium cyanide was used as the colour reagent. Hexamine was determined quantitively by hydrolysing with standard acid and back titrating with standard alkali.

- The results prove conclusively that the products examined possess high degrees of solubility in components of vegetable or animal origin. Noteworthy is the high solubility in lanoline of T.N.T., picric acid, and hexamine. Only in the case of liquid paraffin was a very low solubility in general recorded. For all the bodies examined paraffin wax was also satisfactory, except perhaps in the case of T.N.T. Even in this case the solubility is higher than would be encountered at skin temperature. It follows, therefore, that lanoline might be used in a tetryl barrier but it would be inadmissible in a barrier designed to protect against the other products. Likewise, castor oil would be satisfactory for T.N.T. and picric acid but not for tetryl.

Vegetable Oils, which are the main constituents 
of vanishing creams, are to be avoided in barriers for nitrobodies. In view of their marked contrast in constitution from the fats present in the skin, paraffinoids will not be readily absorbed by the surface. In view of the action of alkali on the skin and on its interaction with nitrobodies to form highly coloured soluble salts which may be toxic, it is considered that the best barrier will be neutral in reaction, at any rate not alkaline.

Attention has already been drawn to the importance of maintaining a film on the skin of about $20 \mu$ thickness, so as to avoid airborne crystalline tetryl of size $5 \mu$ from making contact with skin by piercing the film. Obviously the thickness of film produced must be determined by the viscosity of the cream at the skin temperature. It is, therefore, suggested that the softening point of the cream should be about $22^{\circ} \mathrm{C}$. This latter fact has also some psychological importance. It is held that if protection is to be guaranteed for some hours, then the worker should appreciate that it takes a few minutes to ensure that an even film has been applied to the whole of the exposed skin surface. The film must not be patchy, and a fairly high softening point ensures that the worker will take time to protect himself, particularly if he has confidence in its protective powers.

It is important that a barrier should be capable of removal by the ordinary processes of soap and water. It is suggested, however, that it should not be too easily removed. It should require considerable amounts of hot water and soap. This helps to prevent the transfer of dangerous dust outside the factory into homes where contact may be made with other persons and with food. An emulsifying agent of the water-soluble type must, therefore, be present.

It will be noted that a barrier cream based on paraffinoid compounds will give protection from all the explosive substances mentioned.

\section{The Camraild Barrier}

Based on the above conditions a new barrier was developed and known during the war as "Camraild barrier." It consisted of a mixture of paraffin wax m.p. $135^{\circ} \mathrm{F}$, ( 20 per cent.), ordinary vaseline ( 40 per cent.), lanette wax, as emulsifying agent (10 per cent.), and water (30 per cent.). The mixture is made up as follows.

The paraffin wax, lanette wax, and vaseline are melted together, keeping the temperature as low as possible. The mixture is cooled to $50^{\circ} \mathrm{C}$. and the water, after heating to the same temperature, added with vigorous stirring until the cream is of uniform consistency. Perfume may be added if desired.

The barrier so prepared tends to have a greasy feel, but if applied to the skin in proper amount and well rubbed in it does not prevent use of the hands for normal manual work. In any case over 60 per cent. of the barriers in use during the war were described as greasy (Table 1). But even then, slight greasiness must not be held against a barrier if it can be proved experimentally that it is efficient for the purpose intended.

It will be recalled (Table 1), that only two of the barriers tested gave protection from tetryl or T.N.T. for three hours, the others all failing in one hour. Accordingly, a test was made with Camraild barrier on ten different observers using the simple technique described on p. 238. Having shown that a negative test was obtained in three hours, the test was extended to six hours and in every case a negative result was recorded for both tetryl and T.N.T. When solid material such as zinc oxide, boric acid were incorporated the results were variable and penetration was found in about 50 per cent. of the tests over a three-hour period. Furthermore, these solids tended to give a gritty character to the cream.

\section{Evaluation}

The test already described is not free from criticism. The personal factor cannot be neglected. For this reason the tests were applied to as large a number of observers as possible. Nevertheless, it was considered desirable to place the test on a more scientific basis, in which the same thickness of film would be tested with the same amount of nitrobody under conditions of controlled humidity.

It is obvious that to prepare a film of $20 \mu$ thickness for test is impracticable. It was agreed after experiment that $1 \mathrm{~mm}$. was easily prepared, and although the tests would not bear the same relationship to those obtained from the previous practical test they would at least be comparable. It was also considered desirable to use the same amount of nitrobody and to expose this over the same area. This was done by using a known and constant volume of a solution of the nitrobody in acetone and allowing the solvent to evaporate at atmospheric temperature over a known area. The solution deposited a film of minute crystals so that unit weight per unit area was always constant.

The test was first of all carried out at $30^{\circ} \mathrm{C}$. However, as several of the creams lost their original texture and became quite hard on exposure at this temperature for more than an hour or two, a lower temperature was adopted. To prevent loss of moisture during the test, the creams were kept exposed to an atmosphere of between 90 and 95 per cent. humidity. The reagent used was strong aqueous $\mathrm{KOH}$ and, in order to reduce the possibility of penetration of this into or through the barrier, the time of test was limited to a maximum of one minute. In this new test, the nitrobody penetrates upwards through the barrier.

The procedure adopted is described below, and in Table 3 are given the results applied to some barriers already referred to, as well as to some later products. It will be seen that the results are in general agreement with those reported, and that Camraild barrier is the most efficient protective agent against both T.N.T. and tetryl. 
Penetration Test for Barrier Creams.-A brass ring about 1 in. diameter, 1.5 in. external diameter, and $1 \mathrm{~mm}$. thick is placed on a carefully cleaned ground-glass surface. The inner circumference of the ring is traced with pencil on the glass. The ring is removed and the circular area on plate is covered with $0 \cdot 1 \mathrm{c} . \mathrm{cm}$. of nitrobody solution. The concentrations used in these tests were $5 \mathrm{~g}$. nitrobody to $50 \mathrm{c.cm}$. acetone. The solvent is allowed to evaporate in air. The ring is replaced as before and the mould so formed filled with the cream under test, the surface of the cream being smoothed and levelled with the sides of the ring by means of a straight-edge.

The test equipment is then placed in a chamber kept at between $20^{\circ}$ and $22^{\circ} \mathrm{C}$., the atmosphere in the chamber being maintained at 90 to 95 per cent. humidity by passage of air previously bubbled through wash bottles of warm water, and using a wet-bulb thermometer inside the chamber. The creams are removed periodically and the surface touched with a small drop of 40 per cent. aqueous $\mathrm{KOH}$. The appearance of a pink colour within one minute of applying alkali is taken to indicate penetration of layer by nitrobody. One specimen allows of three to four tests with alkali being carried out. When a positive is obtained, it should be checked on a fresh specimen. That duplication of time of penetration is attainable without difficulty has been established after a large number of observations.

When an emulsified non-absorbable cream is applied to the skin, it may be assumed that $(a)$ water is absorbed by the skin, leaving a film of the actual barrier substance, or $(b)$ water is lost by evaporation from the cream film. In the case of $(b)$, it may well be that the rate of evaporation of water from the film of cream may be compensated by removal of water from the skin to the film, an equilibrium being thus established.
TABLE 3

PENETRATION (IN HOURS) OF T.N.T. AND TETRYL THROUGH BARRIERS $1 \mathrm{MM}$. IN THICKNESS AT 20 TO $22^{\circ} \mathrm{C}$. AT A HUMIDITY OF 90 TO 95 PER CENT.

\begin{tabular}{c|l|l}
\hline Sample & T.N.T. & Tetryl \\
\hline $\mathbf{A}_{2}:$ & pos. 2 & pos. 2.5 \\
$\mathbf{B}_{2}$ & pos. 24 & pos. 20 \\
$\mathrm{C}_{2}$ & pos. 1 & pos. 2 \\
$\mathrm{H}$ & pos. 2 & pos. 2.5 \\
$\mathrm{Q}$ & pos. 6 & pos. 16 \\
$\mathrm{T}$ & pos. 2 & pos. 1 \\
$\mathrm{~W}$ & pos. 3 & pos. 3 \\
Camraild & neg. 24 & neg. 20 \\
\hline
\end{tabular}

Note on Lanoline.-Penetration tests were also carried out on lanoline. To obtain a film of this substance, it was necessary to raise its temperature to $30^{\circ} \mathrm{C}$. and to maintain this temperature during test. No control was kept on the humidity of atmosphere as there should be little loss of moisture from lanoline. The result was : T.N.T., pos. 16 ; tetryl, neg. 16 . This result is in accord with the solubilities of these nitrobodies in lanoline already reported.

The authors desire to thank the Explosives Directorate of the Ministry of Supply for permission to publish this paper.

\section{REFERENCES}

Bedford, T., and Warner, C. G. (1942). J. industr. Hyg., 24, 315. Brownlie, I. A., and Cumming, W. M. (1946). Biochem. J., 40, 20.
Cumming, W. M., and Wright, W. G. D. (1945). Brit. J. industr. Cumming, w, M.,

Sadler, C. G. A.,. and Marriott, R. H. (1946). Brit. med. J., 2, 769.

Swanston, C. (1943). Proe. roy. Soc. Med., 36, 633.

Witkowski, L. J., Fischer, C. N.,, and Murdock, H. D. (1942). J. , Amer. med. Ass., 119, 1406 